EVOLUTIONARY GENETICS

\section{It doesn't have to be...perfect}

If you add up all the ways that cells can go wrong - from DNA mutations and up - it's a small miracle that we function at all. As a mathematical model now shows, there are selective pressures to keep the gene mutation rate as low as possible, but the mistakes that build up en route from gene to protein (the phenotypic mutation rate) are substantially higher and not easy for natural selection to keep in check.

There has been a long-standing interest in measuring the genotypic and phenotypic mutation rate across organisms and in looking at how rates compare. The most striking observation is that although the genotypic mutation rate (whether per base pair, gene or genome) varies widely across organisms and even within species, the phenotypic rate is more or less constant and is several magnitudes higher than the genotypic rate. Why might this be? Surely the cell needs to produce proteins as faithfully as possible and avoid making too many mistakes?

Reinhard Bürger and his colleagues have developed a mathematical model to explore the genotypic and phenotypic rates that could occur in a hypothetical population while still allowing it to function. In their model the population consists of single-celled organisms with a DNA chromosome - the organisms accrue deleterious genotypic and phenotypic mutations at a certain number of genes and the model explores the effect on organismal fitness of different phenotypic or genotypic rates. The fitness costs that a certain mutation rate incurs are measured in relation to how it affects the number of functional proteins that the cell produces.

Surprisingly, but in line with what is observed in nature, the model predicts that populations, even when small, would experience pressure to bring the genotypic mutation rate down to zero (or at least well below $1 \%)$. On the other hand, there is no strong pressure to reduce the phenotypic mutation rate, even in large populations: there is a higher threshold beyond which the phenotypic mutation rate cannot pass, but the lowest threshold value could be as high as $10 \%$. The reason for the large discrepancy between the two rates lies in the different way in which phenotypic and genotypic errors affect an organism's fitness.
The next step will be to apply the parameters to a more realistic scenario; for example by allowing different loci to vary in how how they behave, by considering the possibility that the process of lowering the mutation rate could itself carry a cost, and by including random genetic drift.

Tanita Casci

ORIGINAL RESEARCH PAPER Bürger, R. et al. Why are phenotypic mutation rates much higher than genotypic mutation rates? Genetics $\mathbf{1 7 2}$, 197-206 (2006) WEB SITES

Reinhard Bürger's web page:

http://homepage.univie.ac.at/Reinhard.Buerger Martin Nowak's web page:

http://www.ped.fas.harvard.edu/people/faculty Martin Willensdorfer's web page: http://www.people.fas.harvard.edu/ willensd 